\title{
AN APPROXIMATE ANALYSIS OF MELTING AND FREEZING OF A DRILL HOLE THROUGH AN ICE SHELF IN ANTARCTICA
}

\author{
By CHI TIEN* and Yin-ChaO YeN
}

(U.S. Army Cold Regions Research and Engineering Laboratory, Hanover, New Hampshire 03755 , U.S.A.)

\begin{abstract}
Asstract. An approximate analysis of the processes of melting and freezing of a drill hole, $500 \mathrm{~m}$ depth and $0.15 \mathrm{~m}$ in initial radius, through an ice shelf was made. Results are expressed in graphical form showing the time available for experimentation under the hole as a function of heating duration. It was also found that refreezing has a much slower rate than melting.

Résumé. Une analyse approximative de la fusion et du regel dans un forage a travers une calotte glaciaire dans $r$ Antarctique. Une analyse approximative des processus de fusion et de regel dans un forage de $500 \mathrm{~m}$ de profondeur et de $0,15 \mathrm{~m}$ de diamètre initial a été conduit dans la calotte glaciaire. Les résultats sont exprimés sous forme d'un graphique montrant le temps disponible pour expérimenter dans le trou en fonction de la durée du rechauffement. On a trouvé aussi que le regel est beaucoup plus lent que la fusion.

Zusammenfassunc. Näherungsanalyse des Schmelzens und Gefrierens in einem Bohrloch durch ein Eisschelf in der Antarktis. Der Prozess des Schmelzens und Gefrierens in einem Bohrloch von $500 \mathrm{~m}$ Tiefe und $0,15 \mathrm{~m}$ Anfangsradius durch ein Schelfeis wurde einer genäherten Analyse unterzogen. Die Ergebnisse sind graphisch dargestellt und geben die verfügbare Zeit für Experimente unter dem Loch als Funktion der Heizdauer an. Es zeigte sich ferner, dass das Wiedergefrieren viel langsamer vor sich geht als das Schmelzen.
\end{abstract}

\section{NOMENCLATURE}

$a_{0}$ Initial radius

$a(t) \quad$ Radius

$a^{\star}$ Dimensionless radius $a / a_{0}$

$A$ A factor defined as $\frac{\beta g(\Delta T) a^{3}}{\nu \alpha}\left(\frac{a}{l}\right)$

C Heat capacity

$g$ Gravitational acceleration

$\left(G \tau_{0}\right)$ Grash of number based on initial conditions $\beta g\left(T_{\text {wo }}-T_{\mathrm{m}}\right) a_{0}{ }^{3} / \nu^{2}$

$h$ Heat-transfer coefficient between ice and water

$H$ Ratio of specific to latent heat in ice $\left(C_{\mathbf{i}}\left(T_{\mathrm{m}}-T_{\mathrm{lo}}\right) / L\right)$

$k$ Thermal conductivity

$k_{1}$ A dimensionless factor $0.667 Z^{\frac{1}{3}} \mathcal{N}$ equal to the initial dimensionless melting rate

$l$ Hole depth

$L$ Latent heat of fusion

$L^{\prime}$ Total of latent heat plus heat necessary to warm ice to $T_{\mathrm{m}}$

$N$ A factor defined as $C_{\mathrm{w}} \Delta T_{\mathrm{o}} \rho_{\mathrm{w}} / L^{\prime} \rho_{1}$

$(N u)$ Nusselt number $h a / k$

$(P r)$ Prandtl number $C \mu / k$

$Q$ Strength of line source

$Q^{*}$ Dimensionless line source $Q / \pi k_{\mathrm{w}}\left(T_{\mathrm{wo}}-T_{\mathrm{m}}\right)$

$t$ Time

$t_{1}$ End of heating period

$t_{2}$ Time when the hole radius reaches a critical value

$t^{\star}$ Dimensionless time $\alpha_{\mathrm{w}} t / a_{0}{ }^{2}$

$T$ Temperature

- Professor and Chairman, Department of Chemical Enginecring and Material Sciences, Syracuse University, Syracuse, New York 13210, U.S.A. 


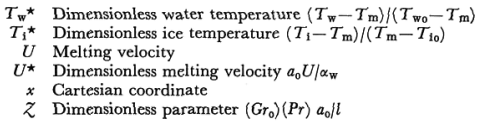

Subscripts

$\begin{aligned} \text { o } & \text { Initial } \\ \text { m } & \text { Melting } \\ \text { w } & \text { Water } \\ \text { i } & \text { Ice } \\ \text { cr } & \text { Critical }\end{aligned}$

Greek letters
a Thermal diffusivity
$\beta$ Coefficient of volumetric expansion
$\delta$ Thermal boundary layer thickness
$\nu$ Kinematic viscosity
$\pi$ Constant
$p$ Density

\section{INTRODUCTION}

The work reported here provides an approximate analysis of the rate of enlargement of a drill hole through an ice shelf, due to the presence of a line heat source (i.e. a heating cable placed inside the hole filled with water) and the subsequent freezing rate upon its removal. The drill hole is $500 \mathrm{~m}$ deep and with an initial radius of $0.150 \mathrm{~m}$. Because of the high closure rate of ice, the drill hole is filled with water during the latter part of drilling. Instrumentation and investigation beneath the hole requires the hole dimension to be maintained above a minimum radius of $0.125 \mathrm{~m}$. In order to provide sufficient time to carry out these measurements, a specified heating cable will be placed throughout its entire depth to enlarge the original drill hole to a certain extent. Thus it is necessary to have some information about the extent of melting as a function of heating duration, and the rate of refreezing upon the removal of the heating cable, as well as the total duration before refreezing has reduced the hole dimension to $0.125 \mathrm{~m}$ in radius. The main objective of this work is to develop a calculation procedure aimed at providing a reasonable estimate of the change of hole dimension due to melting and refreezing under a variety of conditions without the need for excessive numerical computation.

\section{Anatysis}

In generalizing the melting-freezing processes associated with the drill hole, the problem can be considered as follows: A hole of depth $l$ and initial radius of $a_{0}$ is drilled through an ice medium of infinite extent and of initial ice temperature $\tau_{10}$ and is filled with water at temperature $\tau_{\mathrm{wo}}$ as soon as the drilling is completed. Immediately, a line source of constant strength $Q$ (energy per unit length and time) is introduced along the axis of the hole for $0<t \leqslant t_{1}$ and is removed for $t \geqslant t_{\mathrm{r}}$. The problem is to obtain the change of the radius as a function of time and to determine the time $t_{2}$ when the hole radius is reduced to its critical value $a_{\mathrm{er}}=0.125 \mathrm{~m}$. 
The problem investigated here consists of two distinct stages. During the period $0<t<t_{1}$, the hole size increases as a result of melting of the surrounding ice. For $t>t_{1}$, the loss of heat from the melted water to surrounding ice results in refreezing. Accordingly, the problem will be analyzed in terms of a melting followed by a freezing period.

\section{Melting period}

A complete description of the heat transfer problem requires consideration of both the ice and water phases, which are two-dimensional (both axial and radial) and unsteady. Furthermore, in the water phase, the inclusion of free convection with the possible effect of maximum density would make a rigorous solution of the problem extremely tedious and difficult, and for the purpose at hand, this would not be justified. Instead the problem will be greatly simplified by the assumption that the heat transfer across the water-ice interface can be described by the use of a heat-transfer coefficient (which can be estimated from other studies). If $T_{w}$ is the average temperature of the water phase, the macroscopic heat balance for any depth in the water is

$$
Q=\left(\rho_{\mathrm{w}} C_{\mathrm{W}}\right)\left(\pi a^{2}\right) \frac{\mathrm{d} T_{\mathrm{w}}}{\mathrm{d} t}+(2 \pi a)(h)\left(T_{\mathrm{w}}-T_{\mathrm{m}}\right),
$$

where $a$ is the hole radius, $T_{\mathrm{m}}$ the melting temperature and $Q$ the strength of the line source used to increase the water temperature as well as being transferred into the surrounding ice medium. If $T_{\mathrm{m}}$ and $Q$ are taken as constant throughout the depth, the heat transfer problem in the ice will be reduced to a one-dimensional one, in the radial direction only. Furthermore, if the effect of the curvature of the cylindrical bore hole on heat transfer is neglected, the onedimensional heat conduction equation in Cartesian coordinates can be used for the present study (it is believed that the use of this assumption will not cause any significant error; on the other hand, considerable saving in computation is obtained with its use).

For problems in conduction of heat involving melting or freezing, the melting (or freezing) rate is not constant but decreases with the increasing time, and the time dependence of the rate diminishes as time increases. Under such situations, the use of a pseudo-steady-state assumption becomes reasonable. Namely, the temperature distribution obtained with the condition of constant melting rate (i.e. $U=$ constant) can be used to approximate the actual temperature distribution. With the above considerations the temperature distribution in the ice (Carslaw and Jaeger, 1959) and the governing equations of the melting period are given as

$$
\begin{aligned}
\frac{T_{\mathrm{i}}-T_{\mathrm{i} 0}}{T_{\mathrm{m}}-T_{\mathrm{io}}} & =\exp \left[-\frac{U}{\alpha_{\mathrm{i}}}(x-a)\right] \quad \text { for } x \geqslant a(t), \\
T & =T_{\mathrm{w}}(t) \quad \text { for } 0<x<a(t), \\
a(t) & =a_{0}+\int_{0}^{t} U(t) \mathrm{d} t, \\
U & =\frac{\mathrm{d} a}{\mathrm{~d} t}, \\
h\left(T_{\mathrm{w}}-T_{\mathrm{m}}\right) & =\rho_{1} U L^{\prime}, \quad L^{\prime}=L+C_{\mathrm{i}}\left(\mathcal{T}_{\mathrm{m}}-T_{\mathrm{io}}\right), \\
Q & =(2 \pi a)(h)\left(T_{\mathrm{w}}-T_{\mathrm{m}}\right)+\pi a^{2}\left(\rho_{\mathrm{w}} C_{\mathrm{w}}\right) \frac{\mathrm{d} T_{\mathrm{w}}}{\mathrm{d} t},
\end{aligned}
$$

and initial conditions

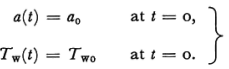


The temperature of the ice and water are given by Equations (2) and (3) respectively if $T_{\mathrm{w}}(t)$ and $\boldsymbol{a}(t)$ are known. These latter quantities can be obtained from a simultaneous solution of Equations (1), (3) and (6) with the initial condition of Equation (7). For this solution, the value of the heat-transfer coefficient $h$ between the water and the ice surface is needed.

The pertinent heat transfer problem in the water phase is not unlike that of the free convection of fluid in a cylindrical container with one open end. A theoretical solution of this was given by Lighthill (1953) for the case of constant surface temperature and fluid temperature at its upper surface. Lighthill's problem is obviously different from the present case. Nevertheless, if the free convection is significant and the water temperature remains more or less uniform and does not vary significantly with time, his results would give a reasonable approximation to the present problem. The average Nusselt number is given as

$$
(\mathcal{N u})=\frac{h a}{k}=0.667 A^{1},
$$

where

$$
A=\frac{\beta g\left(T_{\mathrm{w}}-T_{\mathrm{m}}\right) a_{0}{ }^{3}}{\nu^{2}}\left(\frac{\nu}{\alpha}\right)\left(\frac{a}{a_{0}}\right)^{4}\left(\frac{a_{0}}{l}\right),
$$

where $\beta, \nu$ and $\alpha$ are the volumetric expansion coefficient, kinematic viscosity and thermal diffusivity of water respectively. $l$ is the height of the cylindrical container and in the present problem is equal to the depth of the drill hole. The justification for using Equation (8) is given in the Appendix.

With the introduction of dimensionless variables,

$$
\begin{aligned}
a^{\star} & =a / a_{0}, \\
U^{\star} & =\frac{a_{0}}{\alpha_{\mathrm{w}}} U, \\
t^{\star} & =\frac{\alpha_{\mathrm{w}}}{a_{0}^{2}} t, \\
T_{\mathrm{w}}{ }^{\star} & =\frac{T_{\mathrm{w}}-T_{\mathrm{m}}}{T_{\mathrm{wo}}-T_{\mathrm{m}}}, \\
Q^{\star} & =\frac{Q}{\pi k_{\mathrm{w}} \Delta T_{\mathrm{o}}}, \\
\Delta T_{\mathrm{o}} & =T_{\mathrm{wo}}-T_{\mathrm{m}},
\end{aligned}
$$

Equations (r), (3) and (6) can be rewritten as

$$
\begin{aligned}
a^{\star} & =\mathrm{I}+\int_{0}^{t^{\star}} U^{\star}\left(t^{\star}\right) \mathrm{d} t^{\star}, \\
U^{\star} & =(\mathcal{N u}) \frac{T_{\mathrm{w}}^{\star} N}{a^{\star}}, \\
\frac{\mathrm{d} T_{\mathrm{w}}^{\star}}{\mathrm{d} t^{\star}}+2(\mathcal{N} u) \frac{T_{\mathrm{w}}}{a^{\star}} & =\frac{Q^{\star}}{a^{\star 2}}, \\
\mathcal{N} & =\frac{C_{\mathrm{w}} \Delta T_{\mathrm{o}} \rho_{\mathrm{w}}}{L^{\prime} \rho_{\mathrm{i}}},
\end{aligned}
$$

at

$$
a^{\star}(0)=\mathrm{I}, \quad T_{\mathrm{w}}{ }^{\star}(0)=\mathrm{I} .
$$


The Nusselt number $(\mathcal{N} u)$ introduced in Equation (8), expressed in terms of the dimensionless variables is

with

$$
(N u)=0.667\left(Z^{\frac{1}{*}}\right) a^{\star} T_{w^{\star \frac{1}{2}}}
$$

$$
z=\frac{\left(G r_{0}\right)(\operatorname{Pr}) a_{0}}{l}
$$

where $\left(G r_{0}\right)$ is the Grashof number based on initial conditions.

Combining Equations ( 17$)$ and $(2 \mathrm{I}$ ) yields

$$
U^{\star}=k_{\mathrm{r}} T_{\mathrm{w}}{ }^{\star 5 / 4} \text {, }
$$

where

$$
k_{\mathbf{1}}=(0.667) Z^{\mathfrak{k}} \mathcal{N}
$$

From Equation (16)

$$
U^{\star}=\frac{\mathrm{d} a^{\star}}{\mathrm{d} t^{\star}} .
$$

Substituting Equation (25) into (23), one has

Further differentiation yields

$$
T_{\mathrm{w}}^{\star}=\left(\frac{\mathrm{d} a^{\star} / \mathrm{d} t^{\star}}{k_{\mathrm{r}}}\right)^{0.8} .
$$

$$
\frac{\mathrm{d} T_{\mathrm{w}}{ }^{\star}}{\mathrm{d} t^{\star}}=(0.8)\left(\frac{\mathrm{I}}{k_{\mathrm{I}}^{0.8}}\right)\left(\frac{\mathrm{d} a^{\star}}{\mathrm{d} t^{\star}}\right)^{-0.2} \frac{\mathrm{d}^{2} a^{\star}}{\mathrm{d} t^{\star 2}} .
$$

Substituting Equations (2r), (26) and (27) into Equation (18) and using the relation given in Equations (23) and (24), a second-order differential equation with $a^{\star}$ as the dependent variable is obtained

with

$$
0.8\left(k_{\mathrm{l}}\right)^{-0.8}\left(\frac{\mathrm{d} a^{\star}}{\mathrm{d} t^{\star}}\right)^{-0.2}\left(\frac{\mathrm{d}^{2} a^{\star}}{\mathrm{d} t^{\star 2}}\right)+\frac{2}{\mathcal{N}} \frac{\mathrm{r}}{a^{\star}} \frac{\mathrm{d} a^{\star}}{\mathrm{d} t^{\star}}=\frac{Q^{\star}}{a^{\star 2}}
$$

$$
a^{\star}(\mathbf{0})=\mathbf{I}
$$

and

$$
\left.\frac{\mathrm{d} a^{\star}}{\mathrm{d} t^{\star}}\right|_{\iota^{\star}=0}=(0.667)\left(Z^{\frac{1}{2}}\right) \mathcal{N}=k_{\mathrm{r}} .
$$

Thus the extent of melting can be obtained from the solution of the above initial-value problem. Details of the calculation are given in later sections.

\section{Freezing period}

After a certain heating period (i.e. $t=t_{1}$ or $t^{\star}=t_{\mathrm{I}}{ }^{\star}$ ) the line source is removed from the drill hole. One is interested in estimating the subsequent freezing rate, and in particular, the duration before the hole size reaches to a critical value (i.e. $t=t_{2}$ when $a=a_{\mathrm{cr}}=0.125 \mathrm{~m}$ ). Strictly speaking, freezing would not commence immediately upon the removal of the heating source. In fact, since the water temperature at the end of the heating period (i.e. $T_{\mathrm{w}}\left(t_{1}\right)$ ) will be above $T_{\mathrm{m}}$, one would expect heat transfer from water to surrounding ice to continue until the water temperature dropped to $T_{\mathrm{m}}$. However since $T_{\mathrm{w}}$ is only slightly above $T_{\mathrm{m}}$, as shown in later calculations, this period is expected to be of short duration. For practical purposes, one may assume an instantaneous drop of $T_{\mathrm{w}}$ to that of $T_{\mathrm{m}}$ upon the removal of the heating source. The temperature profile at this point is schematically shown in Figure 1 . 


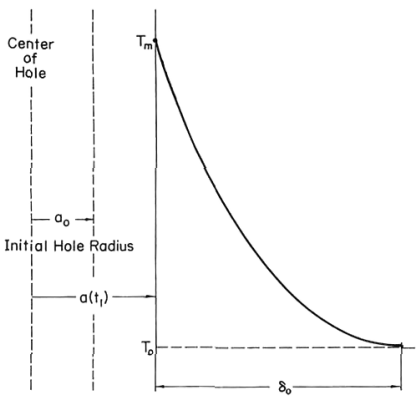

Fig. I. Schematic representation of the ice temperature profile at the end of the heating period.

Due to the existence of the temperature gradient, heat is conducted away radially, which, in turn, necessitates the freezing of water. To estimate the rate of freezing, the heat-balance integral method of Goodman (1958) is used. The basic premise of Goodman's method is based on the physical fact that the significant part of the temperature profile lies only within a restricted neighborhood near the boundary. This region, in the case of one-dimensional conduction, is called the thermal layer whose thickness $\delta$ is, in general, much less than the overall dimension of the medium under consideration. The integral method is used to attempt to obtain an approximate solution by satisfying the conduction equation over this thermal layer on an average basis.

In the present case, the thermal layer refers to the region extending outward from the ice-water interface. This thickness is denoted by $\delta$, then for $x>a+\delta$, the temperature remains undisturbed, i.e. $T_{1}=T_{10}$.

The one-dimensional conduction equation for the ice is given as

$$
\alpha_{i} \frac{\partial^{2} T_{i}}{\partial x^{2}}=\frac{\partial T_{i}}{\partial t} \quad \text { for } t>t_{1}, x>a\left(t_{\mathrm{I}}\right) .
$$

Integrating Equation ( $3 \mathrm{I}$ ) from $x=a$ to $x=a+\delta$ yields

$$
\alpha_{1}\left[\left.\frac{\partial T_{1}}{\partial x}\right|_{a+\delta}-\left.\frac{\partial T_{1}}{\partial x}\right|_{a}\right]=\int_{a}^{a+\delta} \frac{\partial T_{1}}{\partial t} \cdot \mathrm{d} x .
$$

Assume the dimensionless temperature profile in ice can be represented by

$$
T_{\mathrm{i}}{ }^{*}=\frac{T-T_{\mathrm{m}}}{T_{\mathrm{m}}-T_{\mathrm{i}_{0}}}=-\frac{2(x-a)}{\delta}+\frac{(x-a)^{2}}{\delta^{2}} .
$$

It can be seen that the temperature of the ice-water interface is at the melting temperature $\left(\mathcal{T}_{1}{ }^{\star}=0\right)$ and the temperature becomes $T_{1}{ }^{\star}=-\mathrm{I}$ at $x=a+\delta$, namely, the temperature 
outside the thermal layer remains undisturbed. Furthermore $\partial T_{1} \star / \partial x=0$ at $x=a+\delta$, indicating no heat flow beyond the thermal boundary layer.

In terms of the dimensionless temperature, Equation (32) becomes

$$
\alpha_{1}\left[\left.\frac{\partial T_{1}^{\star}}{\partial x}\right|_{a+\delta}-\left.\frac{\partial T_{1}^{\star}}{\partial x}\right|_{a}\right]=\int_{a}^{a+\delta} \frac{\partial T_{1}^{\star}}{\partial t} \mathrm{~d} x .
$$

The right-hand side can be evaluated as follows

$$
\frac{\mathrm{d}}{\mathrm{d} t} \int_{a}^{a+\delta} T_{1}^{\star} \mathrm{d} x=T_{1}^{\star}(a+\delta) \frac{\mathrm{d}(a+\delta)}{\mathrm{d} t}+\int_{a}^{a+\delta} \frac{\partial T_{1}}{\partial t} \mathrm{~d} x-T_{1}^{\star}(a) \frac{\mathrm{d} a}{\mathrm{~d} t} .
$$

Since $T_{1}^{\star}=-\mathrm{I}$ at $x=a+\delta$ and $T_{1}^{\star}=0$, at $x=a$, Equation (35) reduces to

$$
\int_{a}^{a+\delta} \frac{\partial T_{1}}{\partial t} \mathrm{~d} x=\frac{\mathrm{d}(a+\delta)}{\mathrm{d} t}+\frac{\mathrm{d}}{\mathrm{d} t} \int_{a}^{a+\delta} T_{1}^{\star} \mathrm{d} x .
$$

The integral on the right-hand side of the above expression can be evaluated with the use of Equation (32)

$$
\frac{\mathrm{d}}{\mathrm{d} t} \int_{a}^{a+\delta} T_{1}^{\star} \mathrm{d} x=-\frac{2}{3} \frac{\mathrm{d} \delta}{\mathrm{d} t} .
$$

Thus

$$
\int_{a}^{a+\delta} \frac{\partial T_{1}^{\star}}{\partial t} \mathrm{~d} x=\frac{\mathrm{d}(a+\delta)}{\mathrm{d} t}-\frac{2}{3} \frac{\mathrm{d} \delta}{\mathrm{d} t} .
$$

The temperature gradient at $x=a$ is

$$
\frac{\partial T_{i}^{\star}}{\partial x}=-\frac{2}{\delta} .
$$

Substituting Equations ( 36$)$ and (37) into (34), we have

$$
\frac{2 \alpha_{1}}{\delta}=\frac{\mathrm{d} a}{\mathrm{~d} t}+\frac{\mathrm{r}}{3} \frac{\mathrm{d} \delta}{\mathrm{d} t} .
$$

At the ice-water interface, $x=a$, one has

$$
-\left.k_{\mathrm{w}} \frac{\partial T_{1}}{\partial x}\right|_{x=a}=-\rho_{1} L \frac{\mathrm{d} a}{\mathrm{~d} t}
$$

or

$$
-\frac{\mathrm{d} a}{\mathrm{~d} t}=\frac{2 k_{\mathrm{w}}\left(\mathcal{T}_{\mathrm{m}}-T_{\mathrm{i}_{0}}\right)}{\delta \rho_{1} L} .
$$

Combining Equations (38) and (39), one has

$$
\frac{\mathrm{I}}{3} \frac{\mathrm{d} \delta}{\mathrm{d} t}=\frac{2}{\delta}\left[\alpha_{1}+\frac{k_{\mathrm{w}}\left(T_{\mathrm{m}}-T_{\mathrm{io}}\right)}{\rho_{\mathrm{i}} L}\right] .
$$

Integrating Equation (40) with the initial condition, $t=t_{1}, \delta=\delta_{0}$, one has

$$
\begin{aligned}
\left(\frac{1}{12}\right)\left[\delta^{2}-\delta_{0}{ }^{2}\right] & =\left(\alpha_{1}+\frac{k_{\mathrm{w}}\left(T_{\mathrm{m}}-T_{\mathrm{io}}\right)}{\rho_{\mathrm{i}} L} k_{\mathrm{w}}\right)\left(t-t_{\mathrm{1}}\right), \\
\delta & =\left[\delta_{0}{ }^{2}+\mathrm{r} 2\left(\alpha_{\mathrm{i}}+\frac{T_{\mathrm{m}}-T_{\mathrm{i} 0}}{\rho_{\mathrm{i}} L} k_{\mathrm{w}}\right)\left(t-t_{1}\right)\right]^{\frac{1}{2}} .
\end{aligned}
$$


The change of the dimension of the drill hole due to freezing can be obtained by combining Equations (39) and $(4 \mathrm{r})$ as

$$
-\frac{\mathrm{d} a}{\mathrm{~d} t}=\frac{2 k_{\mathrm{W}}\left(T_{\mathrm{m}}-T_{\mathrm{i0}}\right)}{\rho_{1} L} \frac{\mathrm{I}}{\left[\delta_{0}{ }^{2}+12\left(\alpha_{1}+\frac{T_{\mathrm{m}}-T_{\mathrm{io}}}{\rho_{\mathrm{i}} L} k_{\mathrm{w}}\right)\left(t-t_{\mathrm{l}}\right)\right]^{1 !}},
$$

and by simple integration, one has

$$
a_{\mathrm{m}}-a=\frac{k_{\mathrm{w}}\left(T_{\mathrm{m}}-T_{\mathrm{i0}}\right)}{3 \rho_{\mathrm{i}} L\left(\alpha_{\mathrm{i}}+\left[\left(T_{\mathrm{m}}-T_{10}\right) / \rho_{\mathrm{i}} L\right] k_{\mathrm{w}}\right)}\left[\left\{\delta_{0}^{2}+\mathrm{r} 2\left(\alpha_{1}+\frac{T_{\mathrm{m}}-T_{\mathrm{i0}}}{\rho_{\mathrm{i}} L} k_{\mathrm{w}}\right)\left(t-t_{\mathrm{l}}\right)\right\}^{\underline{1}}-\delta_{0}\right]
$$

where $a_{\mathrm{m}}$ is the hole radius at the end of the heating period. In terms of dimensionless variables Equation (42) becomes

where

$$
a_{\mathrm{m}}{ }^{\star}-a^{\star}=\frac{H}{3(\mathrm{r}+H)}\left[\left\{\delta_{0}^{\star 2}+\mathrm{I} 2(\mathrm{I}+H) \frac{\alpha_{1}}{\alpha_{\mathrm{m}}}\left(t^{\star}-t_{1}{ }^{\star}\right)\right\}^{\frac{1}{*}}-\delta_{0}^{*}\right]
$$

$$
H=\frac{C_{\mathrm{i}}\left(T_{\mathrm{m}}-T_{\mathrm{io}}\right)}{L}
$$

and

$$
\delta_{0} \star=\frac{\delta_{0}}{a_{0}} .
$$

Thus one can predict the change of the drill-hole radius after the removal of the heating source provided the value of $\delta_{0}$ is given.

To evaluate $\delta_{0}$, the temperature profile in the ice at $t=t_{\mathrm{r}}$ obtained from Equation (2) has to be matched with that of Equation (33). In other words, one attempts to obtain equivalence between the following two expressions

$$
\frac{T_{\mathrm{i}}-T_{\mathrm{io}}}{T_{\mathrm{m}}-T_{\mathrm{io}}}=\exp \left[-\frac{U\left(t_{\mathrm{I}}\right)}{\alpha_{\mathrm{i}}}\left\{x-a\left(t_{\mathrm{i}}\right)\right\}\right]
$$

and

$$
\frac{T_{1}-T_{\mathrm{m}}}{T_{\mathrm{m}}-T_{\mathrm{io}}}=-2\left(\frac{x-a}{\delta_{0}}\right)+\left(\frac{x-a}{\delta_{0}}\right)^{2} .
$$

It is obvious that these two expressions cannot be made to be equivalent. In an approximate manner, one may require the temperature gradient at the water-ice interface by these two expressions to be the same since it is the ability of ice to conduct heat which determines the rate of freezing,

$$
-\frac{U\left(t_{1}\right)}{\alpha_{1}}=-\frac{2}{\delta_{0}} .
$$

In terms of dimensionless variables, one has

$$
\delta_{0}^{\star}=2\left(\frac{\alpha_{1}}{\alpha_{\mathrm{w}}}\right) \frac{1}{U^{\star}\left(t_{\mathrm{r}}^{\star}\right)} .
$$

Accordingly one can calculate the change of drill-hole radius from Equations (43) or (47) based on the final conditions of the melting period.

\section{Galculation}

As shown in the previous section, for the melting period, the change of drill-hole size is obtained from the solution of Equation (28) with the initial conditions given by Equations (29) and (30). 


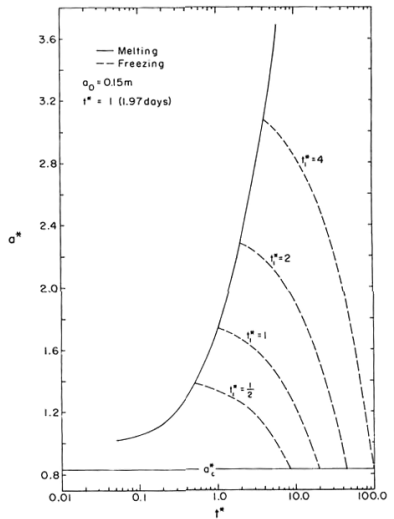

Fig. 2. Dimensionless hole radius $a^{\star}$ as a function of dimensionless time $t^{\star}$ with heating duration $t_{1}^{\star}$ as parameter $\left(a_{0}=0.15 \mathrm{~m}\right)$.

Numerical solutions of Equation (28) were obtained using the Runge-Kutta method. The following conditions and property values were used:

$$
\begin{array}{lc}
\text { Depth of drill hole } & l=500 \mathrm{~m} \\
\text { Initial drill hole radius } & a_{0}=0.15 \mathrm{~m} \\
\text { Melting temperature } & T_{\mathrm{m}}=-2^{\circ} \mathrm{C} \\
\text { Initial ice temperature } & T_{\mathrm{io}}=-20^{\circ} \mathrm{C} \\
\text { Initial water temperature } & T_{\mathrm{wo}}=\mathrm{r}^{\circ} \mathrm{C} \\
\text { Strength of line source } & Q=\mathrm{I} 85 \mathrm{~W} / \mathrm{m} \\
L & =80 \mathrm{kcal} / \mathrm{kg} \\
C_{\mathrm{w}} & =4.185 \mathrm{~kJ} / \mathrm{kg} \mathrm{deg} \\
C_{\mathrm{i}} & =2.092 \mathrm{~kJ} / \mathrm{kg} \mathrm{deg} \\
\rho_{\mathrm{w}} & =\mathrm{r} 000 \mathrm{~kg} / \mathrm{m}^{3} \\
\rho_{\mathrm{i}} & =900 \mathrm{~kg} / \mathrm{m}^{3} \\
k_{\mathrm{w}} & =0.05533 \mathrm{~kJ} / \mathrm{m} \mathrm{deg} \mathrm{s} \\
k_{\mathrm{i}} & =0.22 \mathrm{I} 8 \mathrm{~kJ} / \mathrm{m} \mathrm{deg} \mathrm{s} \\
\Delta T_{\mathrm{o}} & =T_{\mathrm{wo}}-T_{\mathrm{m}}=3 \mathrm{deg} \\
L^{\prime} & =L+C_{\mathrm{i}}\left(T_{\mathrm{m}}-T_{\mathrm{i} 0}\right)=372 \mathrm{~kJ} / \mathrm{kg} \\
\mathcal{N} & =\frac{C_{\mathrm{w}} \rho_{\mathrm{w}}\left(\Delta T_{0}\right)}{L^{\prime} \rho_{\mathrm{i}}}=0.03745
\end{array}
$$




$$
\begin{aligned}
Q^{\star} & =\frac{Q}{\pi\left(\Delta T_{0}\right) k_{\mathrm{w}}}=35.45 \\
Z & =\frac{\left(G r_{0}\right)(P r) a_{0}}{l}=0.7242 \times 10^{5} \\
K_{\mathrm{r}} & =0.667 Z^{\frac{1}{k}} \mathcal{N}=0.4100
\end{aligned}
$$

The results are shown in Figure 2 in which the dimensionless hole size $a^{\star}$ is plotted against $t^{\star}$. The freezing curves at the end of each heating period $t_{1}^{\star}$ are calculated in the following manner: At the end of any particular heating period, a pair of values $a_{\mathrm{m}}{ }^{\star}$, and $U^{\star}$ are computed. The value of $U^{\star}$ is used to estimate the $\delta_{0}$ from Equation (47), and with the given value of $a_{\mathrm{m}} \star$, Equation (43) is used to compute $a^{\star}$ with the given values of $H=C_{1}\left(T_{\mathrm{m}}-T_{\mathrm{i0}}\right) /$ $L=0.1125$ and $\alpha_{1} / \alpha_{w}=8.9$. The change of $a^{\star}$ versus $t^{\star}$ for the freezing period is also shown in Figure 2 on dotted lines each of which correspond to a particular value of $t_{\mathrm{r}}{ }^{\star}$. Figure 3 shows the total time available for experimentation or the length of time when the drill hole reaches the critical radius $a_{\mathrm{er}}=0.125 \mathrm{~m}$ (i.e. the value of $t^{\star}$ for which $a^{\star}=0.125 / 0.150=$ o.833) as a function of heating duration. It gives a straight-line relationship on a double logarithmic plot.

With everything being identical to the case given above but with $a_{0}=0.30 \mathrm{~m}$, and $a_{\mathrm{cr}}=0.25 \mathrm{~m}$, the melting and freezing curves are shown in Figure 4 . The time available for experimentation is also shown in Figure 3. It can be scen that the single line can represent both cases as long as the proper proportional factor is used to convert the dimensionless time to real time.

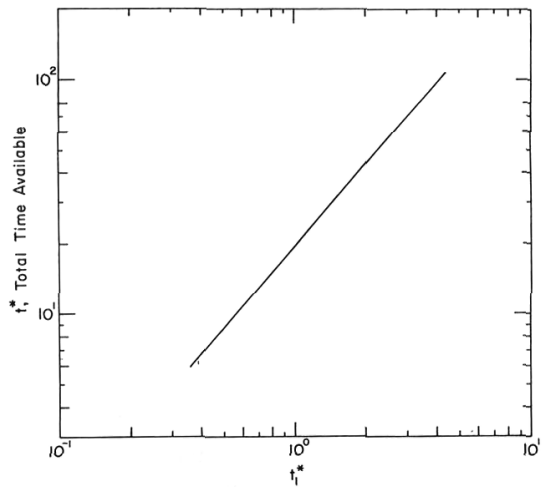

Fig. 3. Total time available for experimentation $t^{*}$ as a function of the heating period $t_{1}^{\star}$ ( for $a_{0}=0.15 \mathrm{~m}$ and $0.30 \mathrm{~m}$ ). 


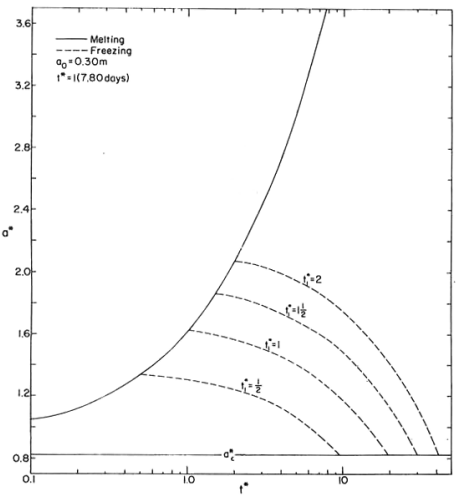

Fig. 4. Dimensionless hole radius $a^{\star}$ as a function of dimensionless time $t^{\star}$ with heating duration $t_{t}^{\star}$ as parameter $\left(a_{0}=0.30 \mathrm{~m}\right)$.

\section{Conclusions}

From the approximate solution it can be concluded that for a specific line heat source, for a dimensionless heating period of $t_{1}{ }^{\star}=0.5$ for example (or $0.985 \mathrm{~d}$ and $3.90 \mathrm{~d}$ of heating for $a_{0}=0.15 \mathrm{~m}$ and $0.30 \mathrm{~m}$ respectively), the time available for conducting experiments under the drill hole is 12.8 and $50.7 \mathrm{~d}$ for $a_{0}=0.15 \mathrm{~m}$ and $0.30 \mathrm{~m}$ respectively. Since the curve is linear on a double logarithmic plot, it can be extrapolated confidently for lower values of $t_{t}{ }^{\star}$. If the duration is longer than needed to accomplish the intended scientific investigations, we need only reduce the heating period or the line strength.

MS. received 20 November 1974 and in revised form $3^{1}$ January 1975

\section{REFERENCES}

Carslaw, H. S., and Jaeger, J. C. 1959. Conduction of heat in solids. Second edition. Oxford, Clarendon Press.

Goodman, T. R. 1958. The heat balance integral and its applications to problems involving a change of phase. Transactions of the American Society of Mechanical Engineers, Vol. 8o, p. 335-42.

Lighthill, M. J. I 953 . Theoretical considerations on free convection in tubes. Quarterly fournal of Mechanics and Applied Mathematics, Vol. 6, Pt. 4, p. 398-439. 


\section{APPENDIX}

\section{JUstification OF USiNg EQUATION (12) FOR THE CALGULATION OF HEAT TRANSFER COEFFicient $h$}

Lighthill (1953) gave a very thorough analysis on the fine convection within a heated vertical tube. He concluded that the flow regimes and subsequently the heat transier can be classified into three categories and are determined solely by the magnitude of the parameter $A$, which is defined as

$$
A=\frac{g \beta(\Delta T) a^{3}}{\nu \alpha}\left(\frac{a}{l}\right) \text {. }
$$

Specifically the three regimes of flow field found are:

(a) $A<\mathrm{I} 000$ : the flow is similar with a stagnant portion at the bottom.

(b) $1000<A<$ to 000 : the flow is of boundary-layer type but not filling the tube.

(c) $A>$ Io ooo: the limiting case becomes identical to the case of a vertical plate.

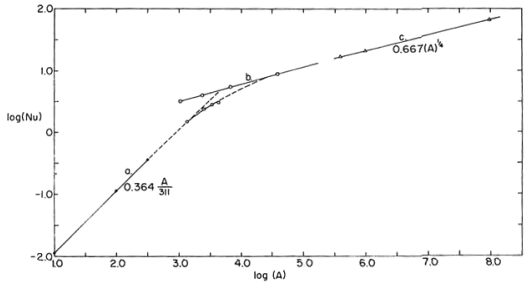

Fig. 5. Heat transfer results of Lighthill's analysis (1953).

The heat transfer result is shown in Figure 5. The Nusselt-number expression of (a) and (c) is also shown in the Figure. The results of (b) are tabulated and no expression is available. A visual obscrvation indicates that an extrapolation of the Nusselt number expression of (c) gives essentially the same values as the tabulated results of (b). Furthermore for the condition considered in this calculation, the corresponding value of the parameter $A$ is always greater than 1 ooo. For this reason the Nusselt number expression of (c) (i.e. Equation (8)) is used in the calculation. 